Boise State University

ScholarWorks

Materials Science and Engineering Faculty

Publications and Presentations

Department of Materials Science and Engineering

$10-1-2007$

\title{
Correlation-Space Description of the Percolation Transition in Composite Microstructures
}

Megan E. Frary

Boise State University

Christopher A. Schuh

Massachusetts Institute of Technology 


\title{
Correlation-space description of the percolation transition in composite microstructures
}

\author{
Megan E. Frary ${ }^{1,2}$ and Christopher A. Schuh ${ }^{1, *}$ \\ ${ }^{1}$ Department of Materials Science and Engineering, Massachusetts Institute of Technology, 77 Massachusetts Avenue, \\ Cambridge, Massachusetts 02139, USA \\ ${ }^{2}$ Department of Materials Science and Engineering, Boise State University, 1910 University Drive, Boise, Idaho 83725, USA
}

(Received 4 June 2007; revised manuscript received 8 August 2007; published 4 October 2007)

\begin{abstract}
We explore the percolation threshold shift as short-range correlations are introduced and systematically varied in binary composites. Two complementary representations of the correlations are developed in terms of the distribution of phase bonds or, alternatively, using a set of appropriate short-range order parameters. In either case, systematic exploration of the correlation space reveals a boundary that separates percolating from nonpercolating structures and permits empirical equations that identify the location of the threshold for systems of arbitrary short-range correlation states. Two- and three-dimensional site lattices with two-body correlations, as well as a two-dimensional hexagonal bond network with three-body correlations, are explored. The approach presented here should be generalizable to more complex correlation states, including higher-order and longer-range correlations.
\end{abstract}

DOI: 10.1103/PhysRevE.76.041108

PACS number(s): 64.60.Ak

\section{INTRODUCTION}

Understanding the structure-property relationship in multiphase materials has been an analytical research goal for many decades [1]. This interest is driven by the need to accurately predict materials properties from microstructural information and, more recently, by the possibility of optimizing one or multiple properties of a composite structure [2-4]. In classical engineering composites, simple geometries or dilute loading fractions have generally permitted reasonable analytical approximations, or at least a series of rigorous bounds on certain properties - notably conductivity, diffusivity, and elastic constants. However, in more complex systems with, e.g., anisotropic phase geometries, nondilute loading levels, and high phase contrast, issues of connectivity and topology become of principal interest. In these situations, the theoretical framework of percolation theory becomes attractive; percolation theory handles phase connectivity naturally through the introduction of the percolation threshold, and complex property scaling in the vicinity of the threshold is captured by a series of simple power-law scaling relations [5-8].

While percolation theory provides at least a semianalytical bridge between complex microstructures and their properties, a recurring issue with its use lies in pinpointing the percolation threshold. This value (which is essentially the phase fraction above which an infinitely connected phase path emerges through the structure) is, unfortunately, generally an empirical constant. While percolation thresholds are tabulated for ideally random phase distributions on different lattice geometries $[9,10]$, any spatial correlations in the phase distribution shift the threshold. For example, geometric phase anisotropy is a common type of spatial correlation in composites. There are many experimental examples in which high aspect-ratio whiskers (e.g., chopped fibers or nanotubes) dramatically lower the percolation threshold as compared with truly random composites [11-15]. Similarly, sys-

\footnotetext{
*schuh@mit.edu
}

tems in which one phase wets the other are also highly correlated and exhibit low thresholds [16-18]. More complex problems involving two- or multiple-body interactions among constituents also exhibit some degree of clustering or ordering and a concomitant shift in the percolation threshold [19-21]. By virtue of shifting the percolation thresholds, spatial correlations also generally disrupt the phase-inversion symmetry enjoyed by randomly distributed systems; i.e., the percolation situation for phase $A$ in a matrix of $B$ is no longer topologically similar to that for phase $B$ in a matrix of $A$.

Although spatial correlations shift the percolation threshold, under the condition that they act only over a small length scale, the scaling relationships of percolation theory are expected to remain valid [22-26]. For example, among the well-known correlated percolation problems in the physics literature [22-25,27-41] the scaling exponents are known to be constant for problems of fixed dimensionality. Such problems include "bootstrap" (and "diffusion") percolation where bonds are randomly assigned but then removed (or added) based on the local environment [23,24] and "sitebond correlated percolation" where neighboring sites are considered to be joined by a bond if both sites are occupied and both of their first neighbor sites along the line of the bond are also occupied [22,27]. Other correlated percolation problems include those studied by Mendelson [28] where a nonrandom distribution is used in assigning site occupancy and geometrical clusters in the random-field Ising model $[29,30,38]$.

The expectation that scaling laws remain valid for shortrange spatial correlations could represent a key advantage of percolation theory for modeling the structure-property relationships of composites. Provided the threshold is known, the variation of properties with changes in phase fraction is captured by the scaling relationships, which are already established for various properties including diffusion, conductivity, and elasticity $[5,11,42-45]$. Thus, in principle, all of the topological complexity of locally correlated systems can be collapsed into a single parameter (or set of parameters) the percolation threshold shift(s) — and property predictions can be made using the scaling relationships. 
Many composite models attempt to describe a composite property directly in terms of spatial correlation information [1]. The alternate method described above, based on cataloging the percolation thresholds of correlated systems, could represent an important complementary approach to the problem. Some authors have made progress in this direction by expressing the percolation threshold in terms of a microstructural state variable for relatively simple microstructure "families." For example, Haan and Zwanzig [46] used a series expansion method to describe phase connectivity in a system of overlapping monosized particles. They examined different local correlation states among the particles, which were described by the average local excluded volume. Lee and Torquato [47] examined the problem of overlapping particles using numerical methods and explicitly calculated the percolation threshold as a function of the allowed degree of overlap. Other authors have used particle shape [48] and size distribution factors [49] as indices of local microstructural correlations and studied the threshold shift as a function of these factors. Heermann and Stauffer [50] explicitly plotted a percolation phase diagram for a site-bond lattice gas percolation problem, using thermodynamic temperature as a state variable.

While the studies described above exemplify the problem and provide useful information about some specific microstructures, they are neither general nor clearly generalizable to arbitrarily correlated microstructures. What is ultimately desired in this context is a description of the percolation threshold in terms of given arbitrary (but physical) spatial correlation information. It is our purpose in this paper to outline an approach to this problem and to take an initial step towards that goal.

\section{METHODOLOGY}

A conventional approach to identify the location of the percolation threshold in a binary system is to run Monte Carlo simulations where the phase fractions $p$ and $q(=1$ $-p$ ) are systematically varied and numerous microstructures are instantiated at each condition. For any given value of $p$, the fraction of lattices that percolate, $\Pi$, is assessed and the threshold is found by identifying the value of $p$ at which $\Pi$ changes from 0 to 1 . For a sufficiently large structure this transition is rapid and finite-size scaling corrections can be made to determine the threshold in the thermodynamic limit. This algorithm is straightforward for lattice structures with random occupation probabilities, because it requires a systematic sweep over just one state variable, $p$.

The general microstructures we are interested in are statistically homogeneous, but exhibit short- (or at least finite-) range correlations; $p$ must be supplemented by detailed correlation information-call it $\mathcal{C}$-in order to uniquely specify the state. Our problem amounts to mapping the percolation threshold as a function of $\mathcal{C}$, requiring that the standard percolation algorithm described above be modified to sweep not only through $p$, but also through $\mathcal{C}$. The difficulty lies in systematically probing the correlation space, which can be quite vast. In the most general case, a complete set of $N$-point correlation functions with anisotropic terms are re- quired and every possible set of such functions must be probed in order to completely map the percolation threshold for arbitrary microstructures. The difficulty is thus one of scope, and accordingly, in order to proceed we will treat only a limited set of possible microstructures (but with an eye toward capturing many practical cases).

The specific binary microstructures we shall consider in this paper satisfy the following conditions: (i) it is possible to digitize the structure onto a regular grid without losing significant information, and (ii) after digitization, the structure contains only nearest-neighbor correlations. The first condition is not limiting per se because given fine enough grid spacing, any microstructure can be described on a discrete lattice. The second condition is limiting, in the sense that there are many microstructures which exhibit correlations beyond the scale of the spatial resolution. However, condition (ii) also dramatically reduces the quantity of correlation information required to simulate every possible microstructure and thus provides a tractable first step. For simplicity, we shall also limit our attention to statistically isotropic microstructures.

Our computational procedure essentially follows the Yeong-Torquato algorithm outlined in Refs. [51-53], which uses a Monte Carlo procedure to swap phase elements of a microstructure in such a way that migrates the system towards a desired configuration. A target set of correlation information is defined, and swaps that bring the system closer to the target are preferentially accepted. In the present case, nearest-neighbor correlation information is used as the set of state variables for the target and the current system configuration. If only selected correlation information is used in this Monte Carlo procedure, the random nature of the algorithm ensures that no independent correlations arise. For example, when the algorithm is used to yield a desired state of shortrange order, no long-range correlations are artificially introduced by the method (beyond emergent order due only to the short-range effects).

We have considered both two- and three-dimensional (2D and 3D) systems and various lattice geometries. In the sections that follow, we discuss several examples of the method and report results mapping the percolation threshold in the correlation space. In the first set of examples, we consider systems in which the correlations are captured entirely by pairwise information among nearest-neighbor elements (i.e., two-body short-range order). In this case a single, conventional short-range order parameter is the only additional state variable required beyond $p$. In the second example we illustrate how the method can be expanded to more complex problems and consider a case where the correlation information requires three-body nearest-neighbor correlation information.

\section{COMPOSITE MICROSTRUCTURES WITH TWO- BODY NEAREST-NEIGHBOR CORRELATIONS}

To investigate of the effect of local correlations on percolation behavior, we begin by considering two-phase composites with only pairwise, nearest-neighbor correlations. Four geometrically unique microstructures were investigated 
(a) $\omega=-1$

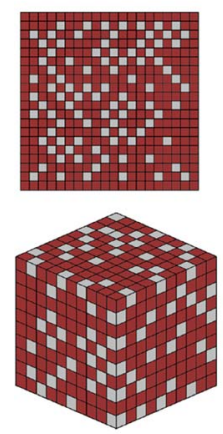

(b) $\omega \rightarrow 1$

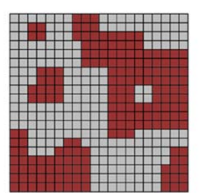

(c) $\omega=1 / 3$

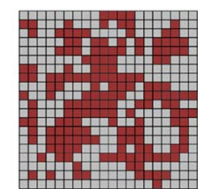

(d) $\omega \rightarrow-1$

(e) $\omega=-1$

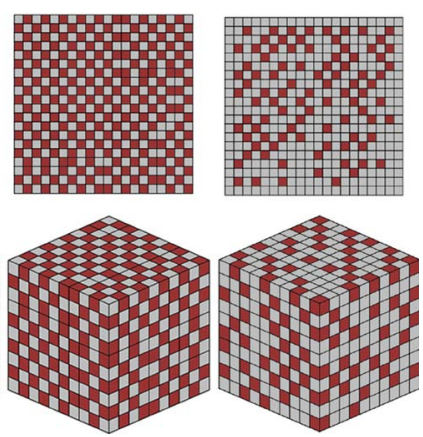

FIG. 1. (Color online) Examples of the two-phase composite microstructures (a)-(e) where the tiles/sites are either squares or cubes and the two phases are shown in red ( $\alpha$ phase) and gray ( $\beta$ phase). The ternary diagram of $D_{i}$ populations is also shown, where the contours correspond to variations in $\omega$ [Eq. (2)]. Ideally segregated structures (with $\omega=1$ ) lie along the edge connecting $D_{0}$ and $D_{2}$, while ideally ordered structures (with $\omega=-1$ ) lie along the other two edges.

where the phases are mapped onto $2 \mathrm{D}$ triangular, square, or hexagonal tiles or onto 3D cubes. In percolation terminology, these are site occupation problems. Some examples of the two-phase composite microstructures are shown in Fig. 1 where the tiles/sites are either squares or cubes and the two phases are shown in dark red ( $\alpha$ phase) and light gray ( $\beta$ phase). In each case a large finite lattice with a characteristic linear dimension of $L=600-1200$ tiles was used.

\section{A. Correlation space}

Simple pairwise correlations may be quantified through $D_{i}$, the fraction of tile pairs which contain $i(=0,1$, or 2$)$ $\beta$-phase tiles. In a randomly assembled composite structure, the distribution of pairs, $D_{i r}$, can be determined as

$$
D_{i r}=\left(\begin{array}{l}
2 \\
i
\end{array}\right)(1-p)^{2-i} p^{i}
$$

where $p$ is the global $\beta$-phase fraction. Spatial correlations in the phase distribution lead to deviations from Eq. (1), but the three values $D_{i}$ are all interdependent by virtue of the fact that (i) they sum to unity and (ii) properly weighted they sum to the $\beta$-phase fraction-i.e., $D_{1}+2 D_{2}=2 p$. Therefore, one way in which the correlation space can be graphically represented is through a ternary diagram of the $D_{i}$, as illustrated in
Fig. 1. Each point in this space uniquely identifies a microstructure (within the assumptions laid out above).

An equivalent approach to quantifying and representing the correlation space is through a scalar short-range order parameter $\omega$, defined in terms of the $D_{i}$ as

$$
\omega= \begin{cases}1-\frac{D_{1}}{D_{1 r}}, & \text { for } \frac{D_{1}}{D_{1 r}} \leqslant 1, \\ \frac{D_{0} D_{2}}{D_{0 r} D_{2 r}}-1, & \text { for } \frac{D_{1}}{D_{1 r}}>1,\end{cases}
$$

where $D_{i r}$ is given in Eq. (1). The order parameter $\omega$, in combination with $p$, fully specifies a microstructure. According to Eq. (2), a perfectly ordered structure has $\omega=-1$ (i.e., all pairs are of the $D_{1}$ type, which resembles a checkerboard pattern on a 2D square lattice), while a perfectly segregated structure has $\omega$ approaching 1 (i.e., all pairs are $D_{0}$ and $D_{2}$ types). The variation in $\omega$ with $D_{i}$ is illustrated in the ternary diagram in Fig. 1 where the vertices represent the population of each pair type. The edge connecting $D_{0}$ and $D_{2}$ corresponds to ideally segregated structures which all have $\omega=1$. The other two edges, connecting $D_{0}$ to $D_{1}$ and $D_{1}$ to $D_{2}$, correspond to ordered structures with $\omega=-1$. The contours indicate how $\omega$ varies from segregated to ordered, and the bold line in the contour plot corresponds to a random composite where $\omega=0$ [i.e., the composites for which Eq. (1) applies]. An example lattice in Fig. 1 can be used to illustrate the procedure for determining the order parameter. In Fig. 1 (a), $D_{0} \approx 0.5, D_{1} \approx 0.5$, and $D_{2}=0$ (i.e., there are no $\beta$ - $\beta$ neighbor pairs). This lattice has $p \approx 0.25$, so Eq. (2) gives $\omega=-1$.

Thus, there are two equivalent representations of the relevant correlation space, one which is represented in the ternary $D_{i}$ diagram of Fig. 1 and a second that may be constructed in the $p-\omega$ plane. Both of these representations are useful, and we shall use both of them in the following discussion. In order to probe the percolation behavior of the two-phase composites described here, random composites were first constructed at a specified value of $p$, and then the Yeoung-Torquato algorithm was used to drive the system toward a particular target in $\omega$. A variety of structures created in this way, and which vary from ordered $(\omega \rightarrow-1)$ to segregated $(\omega \rightarrow 1)$, are shown in Fig. 1. For each structure, we also measured various topological properties of the $\beta$ phase, including the connectivity length $\xi$ and the mean cluster size $S$ of finite clusters, as well as the strength of the "infinite" cluster in percolating systems $P$. We use the standard definitions of these variables (see, e.g., Ref. [9]) and have calculated them for each instantiated microstructure using the Hoshen-Kopelman algorithm [54,55]. Each of these properties can be mapped on to the correlation space, as shown, for example, in Fig. 2 for the connectivity length of the $\beta$ phase on hexagonal lattices. Here both equivalent representations are shown-i.e., the ternary $D_{i}$ space in Fig. 2(a) and the $p$ - $\omega$ space in Fig. 2(b).

Two interesting points emerge from Fig. 2. First, in both figures we can see a band along which the connectivity length diverges. (The infinite or spanning cluster is neglected in the calculation of $\xi$.) This divergence is associated with 

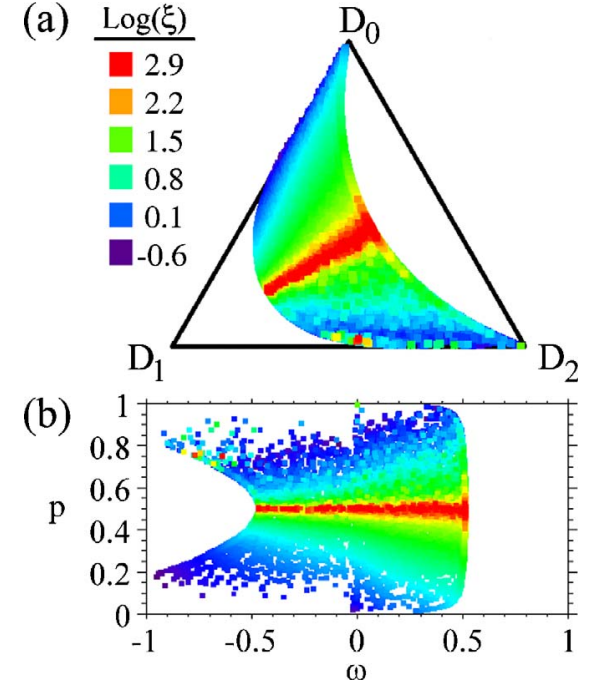

FIG. 2. (Color online) Maps of the connectivity length $\xi$ of the $\beta$ phase on the triangular site lattice in the two representations of the correlation space: (a) ternary $D_{i}$ space or (b) $p$ - $\omega$ space. The connectivity length diverges along a band which corresponds to the percolation threshold.

the percolation threshold and will be analyzed in more detail in the next section. Second, we see that only a portion of the correlation space is accessible with the present simulation procedure. For some lattices, not all combinations of $p$ and $\omega$ or, equivalently, not all triplets $D_{i}$ can be achieved in a composite of finite size that is otherwise random (i.e., without introducing higher-order correlation information). For example, a microstructure with $D_{1}=1$ is not physically possible for hexagonal tiles, although it is for triangular, square, and cubic tiles. On the other hand, some physically possible states are simply inaccessible as an artifact of using a finitesized lattice. For example, a perfectly segregated composite is impossible away from the thermodynamic limit; there must be $D_{1}$ pairs along the interfaces of the segregated regions, and the fraction of $D_{1}$ pairs approaches zero only as the lattice becomes infinitely large. For these reasons, only a portion of the correlation space is accessible using the present procedure.

\section{B. Determination of percolation thresholds}

A more precise determination of the percolation threshold was made by first dividing the correlation space into small bins and calculating the probability of finding a percolating cluster among the many simulated lattice instances in each bin. The percolation threshold was operationally defined as the locus of points where the percolation probability is $50 \%$, as interpolated linearly between the discrete bins. For the lattice sizes used here, this leads to uncertainty of about \pm 0.01 on the location of the threshold in any state variable (e.g., $p$ or $\omega$ ).

Figure 3 summarizes our findings regarding the effects of topological ordering on the position of the percolation threshold in the two complementary (and equivalent) representations of the correlation space. In Fig. 3(a), the percola- (a)
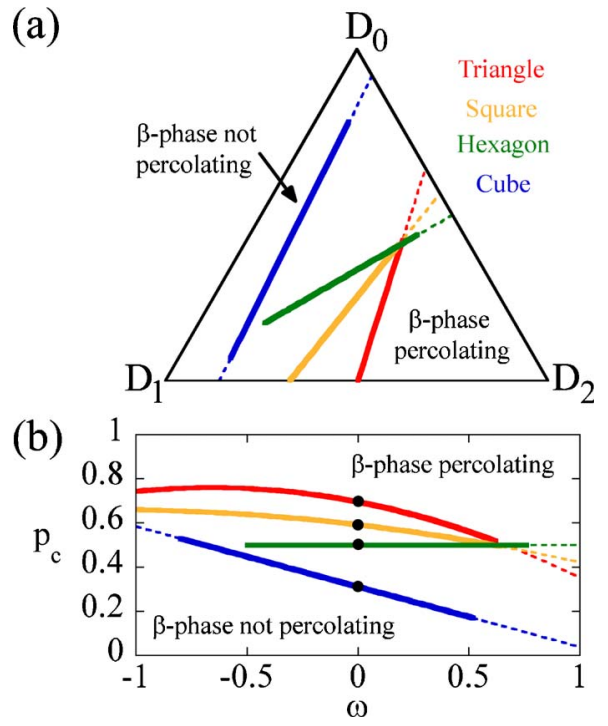

FIG. 3. (Color online) Percolation thresholds for various lattices, represented as (a) a line in the ternary $D_{i}$ diagram or (b) a curve in the $p$ - $\omega$ space. Solid lines indicate states which were explicitly accessed in the present simulations, while dotted lines indicate states that were not accessible due primarily to finite-size effects. In (a), the lines separate correlation states in which the $\beta$ phase percolates (points nearer the $D_{2}$ vertex) from those where it does not (points nearer the $D_{0}$ vertex). In (b), the $\beta$ phase percolates for points which lie above the curve, while the $\beta$ phase does not percolate for points below the curve. The values of $p_{c}$ for $\omega=0$ [black dots in (b)] correspond to those for randomly assembled lattices.

tion thresholds for various lattices are represented as a line in the ternary $D_{i}$ diagram, which separates those portions of the space in which the $\beta$ phase percolates from those where it does not. Composites with a distribution $D_{i}$ lying above the percolation line (i.e., nearer the $D_{0}$ vertex) do not exhibit a percolating $\beta$ phase, while those below the line (i.e., nearer the $D_{2}$ vertex) percolate.

Although it is straightforward to determine the value of $p_{c}$ from Fig. 3(a), it is easier to directly observe the effects of topology in the complementary $p-\omega$ space [Fig. 3(b)]. When $\omega=0$, the value of $p_{c}$ matches exactly the values predicted by standard percolation theory for a random site percolation problem for each of the lattices [9]. Here points which lie above the line correspond to percolating $\beta$-phase networks, while those below the line do not percolate. In both Figs. 3(a) and 3(b), the solid lines extend only over the portion of the correlation space accessible in the present simulations.

There are several interesting conclusions that can be drawn from Fig. 3. To begin, it is evident from Fig. 3(b) that the percolation threshold for hexagonal-tile composites (triangular site lattice) is, to within the accuracy of our calculations, invariant at $p=0.5$ regardless of the short-range topological state of the microstructure. This result is anticipated on the basis of Mendelson's work [28]. Although he considered a different type of spatial correlation, he used a renormalization group approach to show that the percolation threshold was exactly $1 / 2$ for the triangular site lattice (hexagonal tiles in the present work) in the correlated case. For 
TABLE I. Lattice specific coefficients for Eq. (3), which gives the percolation threshold as a function of the correlation state for $2 \mathrm{D}$ and $3 \mathrm{D}$ site lattices. The coefficient $b_{3}$ corresponds to the value of $p_{c}$ for a randomly assembled composite [9,58-60].

\begin{tabular}{ccccc}
\hline \hline & Triangular & Square & Hexagonal & Simple cubic \\
\hline$b_{1}$ & -0.1506 & -0.0515 & 0 & 0 \\
$b_{2}$ & -0.1959 & -0.1190 & 0 & -0.2734 \\
$b_{3}$ & 0.6970 & 0.5927 & 0.5 & 0.3116 \\
\hline \hline
\end{tabular}

other lattices, ordered systems $(\omega<0)$ have higher percolation thresholds than do segregated states $(\omega>0)$, which is reasonable in light of the fact that short-range ordering, by our definition, avoids near-neighbor contacts of like phases. On the other hand, segregation can be very effective in lowering the threshold; for a segregated cube-tiled composite, the percolation threshold approaches a value below $\sim 0.2$ (much lower than $p_{c} \approx 0.31$ for a random cubic lattice). Both of these trends are in line with experimental literature, in which composites with short-range order and repulsion among the reinforcement particles exhibit increased threshold values $[42,56]$, while segregation lowers the threshold [57]. Finally, it should be noted that the curves for hexagonal, triangular, and square tiles appear to cross $p=0.5$ near a single value of $\omega(=2 / 3)$ in Fig. 3(b). The values $p=1 / 2$ and $\omega=2 / 3$ correspond to a tile pair distribution of $D_{0}=5 / 12$, $D_{1}=1 / 6$, and $D_{2}=5 / 12$.

As a tool for practical analysis of simple correlated microstructures, the numerical results in Fig. 3 can be fitted to yield an empirical function that gives the percolation threshold as a function of the correlation state:

$$
p_{c}=b_{1} \omega^{2}+b_{2} \omega+b_{3},
$$

with lattice-specific coefficients given in Table I and implied uncertainty on $p_{c}$ of \pm 0.01 . It should be noted that the coefficient $b_{3}$ corresponds to the known percolation threshold for a randomly assembled composite and is fixed to the latticespecific value given in Refs. [9,58-60].

\section{Scaling behavior}

As noted in the Introduction, sufficiently local correlations shift the percolation thresholds of the composites, but do not change the universality class of the problem; i.e., the critical scaling exponents are unchanged. In this section we briefly examine the connectivity length, mean cluster size, and infinite cluster strength and verify that their scaling behavior obeys this expectation for one example lattice geometry-the hexagonal tile composites. Each of these lattice properties scales with the system's distance from the percolation threshold:

$$
\begin{aligned}
& \xi=C_{\xi}\left|p-p_{c}\right|^{-\nu}, \\
& S=C_{S}\left|p-p_{c}\right|^{-\gamma},
\end{aligned}
$$
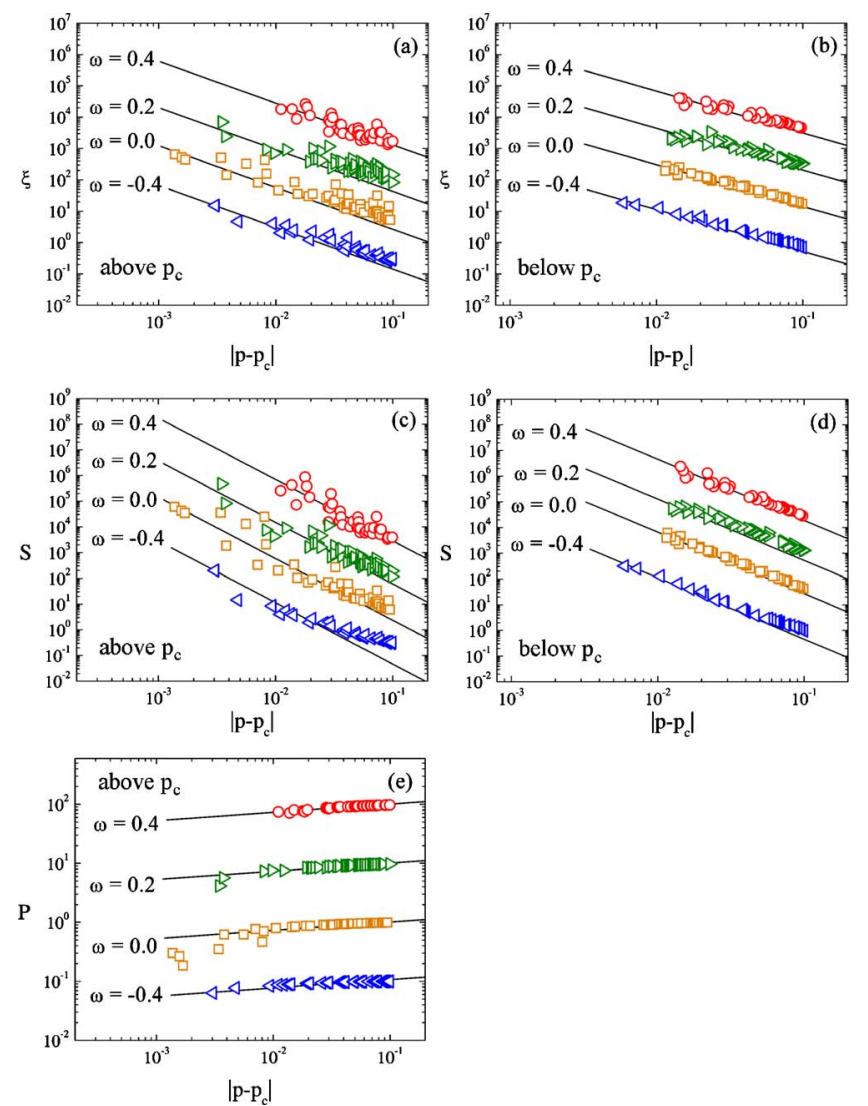

FIG. 4. (Color online) For hexagonally tiled composites (triangular site lattices) with different topological states $(\omega=-0.4,0.0$, 0.2 , and 0.4 ), the lattice properties are plotted as a function of distance from the percolation threshold for values of $p$ above the threshold (a), (c), (e) and below the threshold (b), (d). The series are offset from one another by factors of 10 (with $\omega=0.0$ having no offset), and the best-fit lines in the plots have been forced to the slopes given by Eq. (4).

$$
P=C_{P}\left|p-p_{c}\right|^{\beta},
$$

where $C_{\xi}, C_{S}$, and $C_{P}$ are amplitude prefactors and $\nu, \gamma$, and $\beta$ are scaling exponents which depend only on the dimensionality of the lattice.

For two-dimensional networks, the values of $\nu, \gamma$, and $\beta$ are $4 / 3,43 / 18$, and $5 / 36$, respectively [9], as assessed for random composites-i.e., along the vertical line along $\omega=0$ in Fig. 2(b). Inspecting this figure, it is clear that for different values of $\omega$, the divergence of the connectivity length at the threshold changes; this is a signature that either the scaling exponent $\nu$ or the amplitude prefactor $C_{\xi}$ changes with the state of order in the system. In Fig. 4, we explore the scaling laws of Eq. (4) at a few selected values of $\omega$ for conditions above $(\xi, S$, and $P$ ) and below $(\xi$ and $S)$ the percolation threshold. The data series are offset from one another by factors of 10, and the best-fit lines in the plots have been forced to the slopes given by Eq. (4). For each property, the data have essentially the same slope, independent of $\omega$. As expected, therefore, the short-range correlations in the network do not affect the scaling exponents. 


\section{BOND NETWORKS WITH THREE-BODY NEAREST- NEIGHBOR CORRELATIONS}

In this section we attempt to illustrate how the methodology applied above can be systematically extended to more complex situations. As a complement to the site problems addressed above, here we shall look at a bond lattice-the hexagonal bond lattice in 2D. We will also extend the correlation information to include three-body nearest-neighbor information. In addition to providing an illustrative example, this geometry also connects to an emerging class of percolation problems in polycrystalline materials, where grain boundaries of different atomic structure are treated as phase species and dominate the structure-property relationship [61-70]. For example, grain boundary types with "special" misorientation relationships can have a positive impact on various percolative properties: superconductivity [71-74], diffusivity [75], cracking [67,68], and creep [76] being examples treated quantitatively to date.

\section{A. Definition of correlation functions}

We begin by again defining a distribution to quantify the local connectivity of the microstructure. The coordination number of the hexagonal bond lattice is $Z=3$, so the local distribution of triplets, $J_{i}$, serves an analogous function to $D_{i}$ in the previous example. $\left(J_{i}\right.$ is commonly measured and modeled in the grain boundary literature and is called the triple junction distribution $[77,78]$.) In a randomly assembled bond lattice we have

$$
J_{i r}=\left(\begin{array}{l}
3 \\
i
\end{array}\right)(1-p)^{3-i} p^{i},
$$

where $p$ is the global fraction of $\beta$-phase bonds and the subscript $r$ denotes a random distribution.

In analogy to the ternary diagram used to represent the $D_{i}$ in Fig. 1, a quaternary "coordination tetrahedron" may be used to represent the correlation space for a problem with three-body correlations (Fig. 5). The equivalent representation of the correlation space (in terms of short-range order parameters) must also be expanded to account for the additional information content of the three-body correlation information. For example, we may define two topological order parameters: $\sigma$, which differentiates states of segregation from ordering much as $\omega$ did in our previous example, and $\chi$, which differentiates the tendency for bonds to form either compact or elongated clusters. These are defined as

$$
\begin{gathered}
\sigma= \begin{cases}1-j_{\sigma} & \text { for } j_{\sigma} \leqslant 1, \\
\left(j_{\sigma}\right)^{-1}-1 & \text { for } j_{\sigma}>1,\end{cases} \\
\chi= \begin{cases}1-j_{\chi} & \text { for } j_{\chi} \leqslant 1, \\
\left(j_{\chi}\right)^{-1}-1 & \text { for } j_{\chi}>1,\end{cases}
\end{gathered}
$$

where

$$
j_{\sigma}=\left(\frac{J_{1}+J_{2}}{J_{0}+J_{3}}\right)\left(\frac{J_{0 r}+J_{3 r}}{J_{1 r}+J_{2 r}}\right),
$$
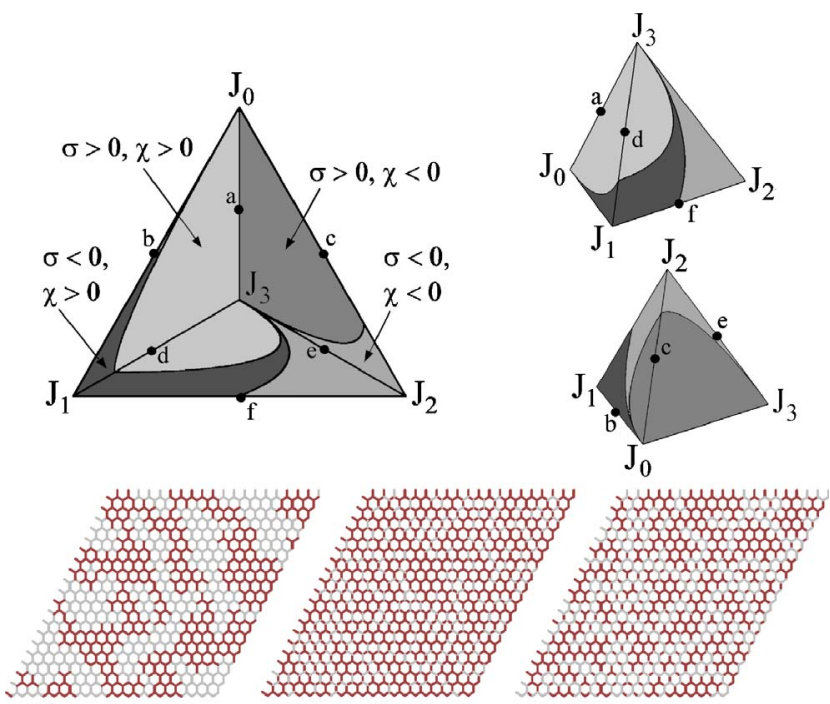

(a) $\sigma \rightarrow 1, \chi \rightarrow 0$

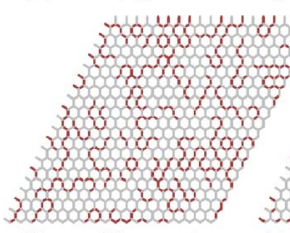

(d) $\sigma \rightarrow 1 / 2, x \rightarrow 1$

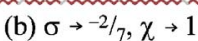

(c) $\sigma \rightarrow 1 / 2, \chi \rightarrow-1$

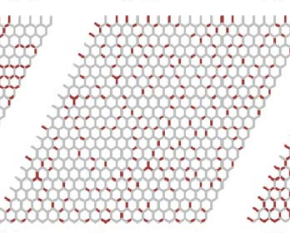

(e) $\sigma \rightarrow-2 / 7, \chi \rightarrow-1$

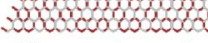

(f) $\sigma \rightarrow-1, \chi \rightarrow 0$
FIG. 5. (Color online) Top: a quaternary "coordination tetrahedron" is used to represent the correlation space for a problem with three-body correlations described by the populations $J_{0}, J_{1}, J_{2}$ and $J_{3}$. The coordination tetrahedron contains four distinct regions where $\sigma$ and $\chi$ [Eqs. (6) and (7)] are independently positive or negative; these four subspaces all converge at the points where $p$ $=0\left(J_{0}\right.$ vertex $)$ and $p=1\left(J_{3}\right.$ vertex $)$. Bottom: several schematic networks are shown that illustrate the various topological states that are possible. In (a)-(f), dark red lines are $\alpha$-phase bonds and light gray lines are $\beta$-phase bonds. The labels (a)-(f) in the top indicate the position of the network in the coordination tetrahedron.

$$
j_{\chi}=\frac{J_{2}}{J_{1}} \frac{J_{1 r}}{J_{2 r}} .
$$

It is useful to apply Eqs. (5)-(7) to an example network to determine the topological order parameters. In Fig. 5(e), nearly all triple junctions are comprised of two or three $\beta$-phase bonds (i.e., $J_{0} \approx 0, J_{1} \approx 0, J_{2} \approx 0.5$, and $J_{3} \approx 0.5$ ). This lattice has $p \approx 5 / 6$, and use of Eqs. (6) and (7) gives $\sigma=-2 / 7$ and $\chi=-1$.

Both $\sigma$ and $\chi$ exist on $(-1,1)$ and are exactly zero for a randomly assembled network. Since the two topological parameters have independent signs, there are four possible combinations for the signs of $\sigma$ and $\chi$, which represent topologically unique states: the network can be composed of either compact or elongated clusters, which may be arranged in either an ordered or segregated fashion. Figure 5 schematically lays out these possibilities, with reference to the position that each network structure occupies in the tetrahedral $J_{i}$ space. Each of the four sign combinations for $\sigma$ and $\chi$ occupies its own subspace, and these four subspaces all converge at the points where $p=0\left(J_{0}\right.$ vertex $)$ and $p=1\left(J_{3}\right.$ vertex $)$. 
Interestingly, this implies that there exist trajectories from $p=0$ to 1 which lie exclusively in each of the four subspaces. Figure 5 also shows several schematic networks that illustrate the various topological states that are possible. For example, points along the $J_{0}-J_{3}$ edge in Fig. 5 describe a segregated structure as shown in Fig. 5(a) for a network with $\sigma \approx 1$. In contrast, a network described by a point along the $J_{1}-J_{2}$ edge assumes an ordered structure with $\sigma \approx-1$ [Fig. $5(\mathrm{f})$ ]. The prominence of $J_{1}$ triplets, especially in points near the $J_{0}-J_{1}-J_{3}$ face, tends to promote compact cluster structures $(\chi>0)$; two examples with $\chi \approx 1$ are illustrated in Figs. 5(b) and 5(d). In contrast, an abundance of $J_{2}$ triplets on the $J_{0}-J_{2}-J_{3}$ face leads to elongated cluster structures. Figure 5 also shows two networks with $\chi \approx-1$ [Figs. 5(c) and 5(e)].

\section{B. Determination of the percolation boundary}

In our example focusing on two-body correlations, states which percolated were separated from states which did not percolate by a curve in either the two-dimensional $D_{i}$ space or the $p-\omega$ space (Fig. 3). The extension to three-body interactions renders the percolation boundary a surface cutting through either the coordination tetrahedron of $J_{i}$ or the $p-\sigma-\chi$ space. Once again, we employed the Yeong-Torquato simulated annealing approach to systematically probe this space. The coordination tetrahedron was divided into bins 0.01 in width along $J_{0}, J_{1}, J_{2}$, and $J_{3}$, each simulation yielding a network lying in one bin. The fraction of networks which contained a percolating cluster was determined, and the surface of $50 \%$ percolation probability was again operationally defined as the percolation condition. It is important to note again that some portions of the correlation space were inaccessible to the algorithm due to, e.g., finite-size effects.

Figure 6 shows representations of the percolation boundary in the two equivalent forms of the correlation space. In both Figs. 6(a) and 6(b), the upper portion of the diagram represents the structures in which the $\beta$-phase bonds percolate. To within the uncertainty of the simulations (and bearing in mind that the edges of the correlation space are sometimes inaccessible) the data suggest that the points $J_{2}=1$ and $J_{0}=J_{3}=\frac{1}{2}$ may lie on the percolation surface. The effect of variations in the network topology on the percolation surface can be more easily visualized in Fig. 6(b), where changes in $p_{c}$ with $\chi$ and $\sigma$ are plotted. There are points within the volume of Fig. 6(b) which do not correspond to a physical combination of triplets (i.e., $0 \leqslant J_{i} \leqslant 1$ for all $i$ ); the percolation boundary presented in Fig. 6(b) is shown only for physically attainable microstructures. As $\chi$ approaches -1 , the percolation threshold decreases, while $\chi>0$ requires an increase in $p$ for percolation to occur; more elongated clusters promote earlier percolation. Similar to our earlier observations in the site-lattice problem, the percolation threshold is lower for $\sigma>0$ where the network is segregated and higher where it is ordered. These trends can also be seen more clearly by looking at the cross-sectional views of the $p-\sigma-\chi$ space in Fig. 7. Here the connectivity length is plotted for two orthogonal sections through the space and the band of high connectivity length denotes the percolation threshold.

Finally, an empirical fit gives the approximate location of the threshold as a function of the correlation state as
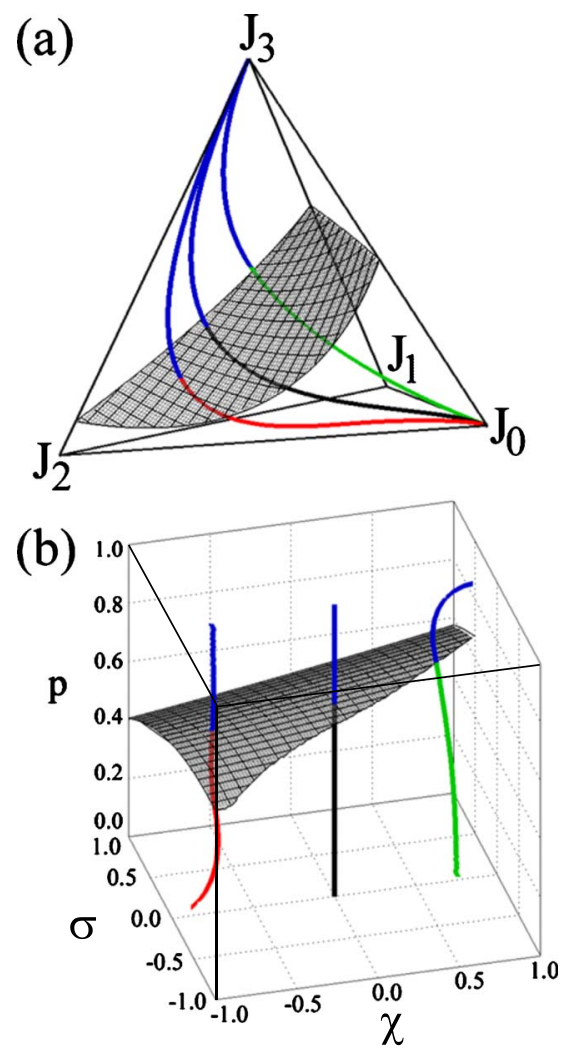

FIG. 6. (Color online) Representations of the percolation boundary in (a) the coordination tetrahedron and (b) the $p-\sigma-\chi$ space. In both, the upper portion of the diagram represents the structures in which the $\beta$-phase bonds percolate. In (b), the percolation boundary is shown only for points which correspond to a physical combination of triplets (i.e., $0 \leqslant J_{i} \leqslant 1$ for all $i$ ). Three trajectories are also shown which correspond to microstructures with systematically varied textures whose thresholds were found previously [62]. The color change in each trajectory represents the percolation threshold which coincides with the surface found here.

$$
p_{c}=d_{1}+d_{2} \chi+d_{3} \chi^{2}-d_{4} \sigma+d_{5} \chi \sigma-d_{6} \sigma^{2},
$$

with the coefficients assembled in Table II. Note that the value of $d_{1}$ has been fixed to the known result of $p_{c}=1$ $-2 \sin (\pi / 18) \approx 0.6527$ for the random hexagonal bond lattice [9].

\section{Connection to the literature on grain boundary networks}

In the literature addressing percolation problems on grain boundary networks, crystallographic textures are assigned to the grains of a polycrystal and the phase character of the grain boundaries is subsequently determined based on, e.g., misorientation of the crystals meeting at the boundary $[61-63,66,67]$. Even in the absence of orientation correlations among the grains, the granular nature of the solid requires correlations in the character of the boundaries between them. Consequently, a single crystallographic texture creates a single statistical microstructure occupying a single point in the correlation space. By systematically varying the texture, different correlation states on the bond lattice (grain boundary network) are effected. In the representations shown in 

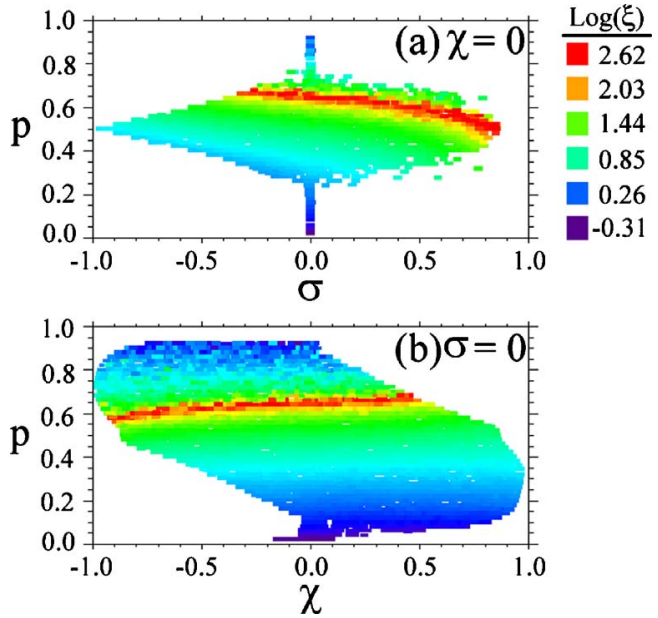

FIG. 7. (Color online) Partial maps of the connectivity length for two-dimensional bond percolation on the hexagonal lattice, with three-body nearest-neighbor correlations. Two cross-sectional views of the $p-\sigma-\chi$ space are shown: (a) $\chi=0$ and (b) $\sigma=0$. The connectivity length diverges along a band which corresponds to the percolation threshold.

Fig. 6, varying the texture function is equivalent to drawing a trajectory through the correlation space. For example, very sharp textures often give $p=1$ and broadening the texture function leads to a smooth trajectory eventually connecting to $p=0$.

In Fig. 6, three trajectories are shown. The first is that for a randomly assembled lattice, as given by Eqs. (5) and (6); in the $p-\sigma-\chi$ space [Fig. 6(b)], this is simply a vertical line where $\sigma=\chi=0$ for all $p$. The other trajectories are the two phase-inversion complements of a typical crystallographically constrained system based on a family of 2D crystal textures labeled as the "fiber texture" family [61]. This family of textures is relevant to the present discussion because in this case crystallography induces strong near-neighbor threebody correlations, but does not have much impact at longer length scales [64]. In Fig. 6 it is clear that these trajectories deviate significantly from that of a randomly assembled lattice for all $p$.

The color change in each trajectory in Fig. 6 represents the percolation threshold (available in Ref. [62]. for the crystallographic systems). All threshold locations coincide well with the percolation surface found in the present work and are within the cited error of the value returned by Eq. (8). This therefore comprises one instance where the dominant physical correlations can be succinctly captured in a small space of order parameters and used to appreciate a problem of practical interest. We expect that other systems of 2D crystallographic texture may be similarly approachable with the present method. In the more general case of 3D textures additional correlations are known to arise at larger length scales [64] and these correlations would need to be added to the correlation space in order for the present approach to be generally applicable.

\section{CONCLUSIONS}

Although finite-range correlations are known to shift the percolation threshold in composite microstructures, a general
TABLE II. Lattice specific coefficients for Eq. (8), which gives the percolation threshold as a function of the correlation state for the honeycomb bond lattice. The coefficient $d_{1}$ corresponds to the value of $p_{c}$ for a randomly assembled hexagonal bond lattice [9].

\begin{tabular}{ccccccc}
\hline \hline$i$ & 1 & 2 & 3 & 4 & 5 & 6 \\
\hline$d_{i}$ & 0.6527 & 0.0651 & 0.0117 & -0.0961 & 0.0233 & -0.0715 \\
\hline \hline
\end{tabular}

framework permitting estimation of the threshold for arbitrary correlations is lacking. We propose that this problem may be addressed systematically by instantiating various correlation states in simulated microstructures and determining the threshold location as a function of the correlations. The reason that this problem has not been approached in a general fashion to date is almost certainly the issue of scope: systematic exploration of correlations over many scales and including anisotropic terms would be a large undertaking. In this paper we have taken a first step towards this goal by exhaustively studying short-range correlated microstructures on a few important lattices. In particular, we report numerical estimates of the percolation threshold for two-dimensional square, hexagonal, and triangular site lattices, as well as three-dimensional cube lattices, with arbitrary (but physically realizable) nearest-neighbor correlations. We also provide results for a two-dimensional hexagonal bond network in which the nearest-neighbor correlations are of the threebody type.

By defining a "correlation space" in which all short-range correlated microstructures exist, it is possible to identify the percolation threshold as a boundary dividing the space into a region in which all microstructures percolate and one in which they do not. The correlations can be described using bond fractions, or appropriate order parameters may be defined; such order parameters are independent indices of the correlations in the system and complement the phase fraction $p$ as state variables. We anticipate that these results may be useful in providing a connection between microstructure and properties of some simple correlated composite structures. One physical example where short-range correlations are sufficient to treat a physically relevant problem is provided - the emerging class of percolation problems on grain boundary networks.

With the percolation thresholds $p_{c}$ provided in this work and existing estimates for the property scaling exponents with distance from the threshold, $\left|p-p_{c}\right|$, improved property predictions should be possible, especially for microstructures close to the threshold. Finally, we suggest that the basic approach taken in this paper may be extended to more complex (and more physically realistic) correlation states by systematically increasing the dimensionality of the correlation space.

\section{ACKNOWLEDGMENT}

This work was supported by the U.S. National Science Foundation under Contract No. DMR-0346848. 
[1] S. Torquato, Random Heterogeneous Materials (SpringerVerlag, New York, 2001).

[2] S. R. Kalidindi, M. Binci, D. Fullwood et al., Acta Mater. 54, 3117 (2006).

[3] S. Torquato, S. Hyun, and A. Donev, Phys. Rev. Lett. 89, 266601 (2002).

[4] S. Torquato, S. Hyun, and A. Donev, J. Appl. Phys. 94, 5748 (2003).

[5] A. Bunde, W. Dieterich, and E. Roman, Phys. Rev. Lett. 55, 5 (1985).

[6] D. Stauffer, Phys. Rep. 54, 1 (1979).

[7] G. Grimmett, Percolation (Springer, Berlin, 1999).

[8] M. Sahimi, Applications of Percolation Theory (Taylor \& Francis, London, 1994).

[9] D. Stauffer and A. Aharony, Introduction to Percolation Theory (Taylor \& Francis, London, 1992).

[10] P. H. L. Martins and J. A. Plascak, Phys. Rev. E 67, 046119 (2003).

[11] Y. P. Mamunya, Y. V. Muzychenko, P. Pissis et al., Polym. Eng. Sci. 42, 90 (2002).

[12] J. N. Coleman, S. Curran, A. B. Dalton, A. P. Davey, B. McCarthy, W. Blam, and R. C. Barklic, Phys. Rev. B 58, R7492 (1998).

[13] R. Murphy, V. Nicolosi, Y. Hernandez et al., Scr. Mater. 54, 417 (2006).

[14] J. C. Grunlan, A. R. Mehrabi, M. V. Bannon et al., Adv. Mater. (Weinheim, Ger.) 16, 150 (2004).

[15] Y. Brechet, J. Y. Y. Cavaille, E. Chabert et al., Adv. Eng. Mater. 3, 571 (2001).

[16] K. M. Golden, Ann. Glaciol. 33, 28 (2001).

[17] K. M. Golden, Physica B 338, 274 (2003).

[18] K. M. Golden, S. F. Ackley, and V. I. Lytle, Science 282, 2238 (1998).

[19] A. Sadiq and M. A. Khan, Z. Phys. B: Condens. Matter 39, 131 (1980).

[20] L. Flandin, T. Prasse, R. Schueler, K. Schulte, W. Bauhofer, and J. Y. Cavaille, Phys. Rev. B 59, 14349 (1999).

[21] F. Carmona and J. Ravier, Physica B 338, 247 (2003).

[22] P. R. A. Campos, L. F. C. Pessoa, and F. G. Brady Moreira, Phys. Rev. B 56, 40 (1997).

[23] C. M. Chaves and B. Koiller, Physica A 218, 271 (1995).

[24] M. C. Medeiros and C. M. Chaves, Physica A 234, 604 (1997).

[25] S. Prakash, S. Havlin, M. Schwartz, and H. E. Stanley, Phys. Rev. A 46, R1724 (1992).

[26] R. F. Voss, R. B. Laibowitz, and E. I. Allessandrini, Phys. Rev. Lett. 49, 1441 (1982).

[27] N. S. Branco and K. D. Machado, Phys. Rev. B 47, 493 (1993).

[28] K. S. Mendelson, Phys. Rev. E 61, 2432 (2000).

[29] L. Kornyei and F. Igloi, Phys. Rev. E 75, 011131 (2007).

[30] E. T. Seppälä and M. J. Alava, Phys. Rev. E 63, 066109 (2001)

[31] R. Thomson, L. E. Levine, and D. Stauffer, Physica A 283, 307 (2000).

[32] P. Grassberger, J. Phys. A 22, 3673 (1989).

[33] M. A. Knackstedt, M. Sahimi, and A. P. Sheppard, Phys. Rev. E 61, 4920 (2000).

[34] M. A. Knackstedt, M. Sahimi, and A. P. Sheppard, Phys. Rev. E 65, 035101(R) (2002).
[35] U. Bauerschafer and M. Schulz, Phys. Rev. E 54, 1442 (1996).

[36] A. Coniglio, H. E. Stanley, and W. Klein, Phys. Rev. Lett. 42, 518 (1979).

[37] M. B. Isichenko, Rev. Mod. Phys. 64, 961 (1992).

[38] C.-K. Hu, Phys. Rev. B 29, 5103 (1984).

[39] J. N. Roberts and L. M. Schwartz, Phys. Rev. B 31, 5990 (1985).

[40] A. Weinrib, Phys. Rev. B 29, 387 (1984).

[41] D. E. Sanders and J. W. Evans, Phys. Rev. A 38, 4186 (1988).

[42] C. Pecharroman, S. Lopez-Esteban, J. F. Bartolome et al., J. Am. Ceram. Soc. 84, 2439 (2001).

[43] A. Kapitulnik and G. Deutscher, Phys. Rev. Lett. 49, 1444 (1982).

[44] K. Yoshida and S. Ueda, Mater. Sci. Res. Int. 1, 114 (1995).

[45] K. Nozaki and T. Itami, J. Phys.: Condens. Matter 16, 7763 (2004).

[46] S. W. Haan and R. Zwanzig, J. Phys. A 10, 1547 (1977).

[47] S. B. Lee and S. Torquato, Phys. Rev. A 41, 5338 (1990).

[48] W. Xia and M. F. Thorpe, Phys. Rev. A 38, 2650 (1988).

[49] A. B. Khanikaev, A. B. Granovskii, and J. P. Clerc, Phys. Solid State 44, 1611 (2002).

[50] D. W. Heermann and D. Stauffer, Z. Phys. B: Condens. Matter 44, 339 (1981).

[51] C. L. Y. Yeong and S. Torquato, Phys. Rev. E 58, 224 (1998).

[52] C. L. Y. Yeong and S. Torquato, Phys. Rev. E 57, 495 (1998).

[53] N. Sheehan and S. Torquato, J. Appl. Phys. 89, 53 (2001).

[54] J. Hoshen, M. W. Berry, and K. S. Minser, Phys. Rev. E 56, 1455 (1997).

[55] J. Hoshen and R. Kopelman, Phys. Rev. B 14, 3438 (1976).

[56] Y. Song, T. W. Noh, S. I. Lee, and B. R. Gaines, Phys. Rev. B 33, 904 (1986).

[57] Y. P. Mamunya, V. V. Davydenko, P. Pissis et al., Eur. Polym. J. 38, 1887 (2002).

[58] P. N. Suding and R. M. Ziff, Phys. Rev. E 60, 275 (1999).

[59] M. E. J. Newman and R. M. Ziff, Phys. Rev. Lett. 85, 4104 (2000).

[60] H. G. Ballesteros, L. A. Fernandez, V. Martin-Mayor et al., J. Phys. A 32, 1 (1999).

[61] M. Frary and C. A. Schuh, Phys. Rev. B 69, 134115 (2004).

[62] M. Frary and C. A. Schuh, Philos. Mag. 85, 1123 (2005).

[63] D. T. Fullwood, J. A. Basinger, and B. L. Adams, Acta Mater. 54, 1381 (2006).

[64] C. A. Schuh and M. Frary, Scr. Mater. 54, 1023 (2006).

[65] C. A. Schuh, M. Kumar, and W. E. King, Acta Mater. 51, 687 (2003).

[66] C. A. Schuh, R. W. Minich, and M. Kumar, Philos. Mag. 83, 711 (2003).

[67] Clinton D. Van Siclen, Phys. Rev. B 73, 184118 (2006).

[68] E. S. McGarrity, P. M. Duxbury, and E. A. Holm, Phys. Rev. E 71, 026102 (2005).

[69] B. W. Reed and M. Kumar, Scr. Mater. 54, 1029 (2006).

[70] A. J. Schwartz, W. E. King, and M. Kumar, Scr. Mater. 54, 963 (2006).

[71] M. Frary and C. A. Schuh, Appl. Phys. Lett. 83, 3755 (2003).

[72] A. Goyal, E. D. Specht, Z. L. Wang et al., Ultramicroscopy 67, 35 (1997).

[73] R. Haslinger and R. Joynt, Phys. Rev. B 61, 4206 (2000). 
[74] B. Zeimetz, B. A. Glowacki, and J. E. Evetts, Eur. Phys. J. B 29, 359 (2002).

[75] Y. Chen and C. A. Schuh, Acta Mater. 54, 4709 (2006).

[76] Y. Chen and C. A. Schuh, Phys. Rev. Lett. 98, 035701 (2007).
[77] M. Kumar, W. E. King, and A. J. Schwartz, Acta Mater. 48, 2081 (2000).

[78] R. W. Minich, C. A. Schuh, and M. Kumar, Phys. Rev. B 66, 052101 (2002). 